This item was submitted to Loughborough's Research Repository by the author.

Items in Figshare are protected by copyright, with all rights reserved, unless otherwise indicated.

\title{
Comparison of two novel MRAS based strategies for identifying parameters in permanent magnet synchronous motors
}

\section{PLEASE CITE THE PUBLISHED VERSION}

http://dx.doi.org/10.1007/s11633-010-0535-3

\section{PUBLISHER}

(C) Springer

VERSION

AM (Accepted Manuscript)

\section{PUBLISHER STATEMENT}

This work is made available according to the conditions of the Creative Commons Attribution-NonCommercialNoDerivatives 4.0 International (CC BY-NC-ND 4.0) licence. Full details of this licence are available at: https://creativecommons.org/licenses/by-nc-nd/4.0/

\section{LICENCE}

CC BY-NC-ND 4.0

\section{REPOSITORY RECORD}

Liu, Kan, Qiao Zhang, Zi-Qiang Zhu, Jing Zhang, An-Wen Shen, and Paul Stewart. 2019. "Comparison of Two Novel MRAS Based Strategies for Identifying Parameters in Permanent Magnet Synchronous Motors". figshare. https://hdl.handle.net/2134/25042. 


\title{
Comparison of Two Novel MRAS Strategies for Identifying
}

\section{Parameters in Permanent Magnet Synchronous Motors}

\author{
Kan Liu $^{1,3^{*}}$ Q. Zhang ${ }^{2,3}$ Z.Q. Zhu ${ }^{3}$ J. Zhang ${ }^{1}$ A.W. Shen ${ }^{2} \quad$ P. Stewart ${ }^{3,4}$ \\ ${ }^{1}$ School of Electrical and Information Engineering, Hunan University, Changsha, 410082, PRC \\ ${ }^{2}$ Department of Control Science and Engineering, Huazhong University of Science and Technology, Wuhan, 430074, PRC \\ ${ }^{3}$ Department of Electronic and Electrical Engineering, University of Sheffield, Sheffield, S1 3JD, UK \\ ${ }^{4}$ Department of Aeronautical and Aut omotive Engineering, University of Salford, UK
}

\begin{abstract}
Two Model Reference Adaptive System (MRAS) estimators are developed for identifying the parameters of permanent magnet synchronous motors (PMSM) based on Lyapunov stability theorem and Popov stability criterion, respectively. The proposed estimators only need online detection of currents, voltages and rotor rotation speed, and are effective in the estimation of stator resistance, inductance and rotor flux-linkage simultaneously. Their performances are compared and verified through simulations and experiments. It shows that the two estimators are simple and have good robustness against parameter variation and are accurate in parameter tracking. However, the estimator based on Popov stability criterion, which can overcome the parameter variation in a practical system, is superior in terms of response speed and convergence speed since there are both proportional and integral units in the estimator in contrast to only one integral unit in the estimator based on Lyapunov stability theorem. In addition, there is no need of the expert experience which is required in designing a Lyapunov function.
\end{abstract}

Keywords: PMSM, MRAS, Ly apunov stability theorem, Popov stability criterion, parameter identification.

\section{Introduction}

${ }^{1}$ Recently, permanent magnet synchronous motors (PMSM) are widely used in high performance servo and other industrial applications due to their high power density and efficiency. However, the power density and control performance suffer from the variation of parameters due to the temperature rise and magnetic saturation etc. Hence, many strategies, such as Neural Network and Extended Kalman filter (EKF) etc. have been proposed to identify the PMSM parameters. However, these strategies are not always reliable in complex industrial application due to the nonideal working condition, and controller design without the consideration of robustness, as will be discussed as follows.

(1) Based on Neural Networks (NN): This strategy is usually based on establishing a NN mathematical model transferred from PMSM current model and the tracking algorithm is usually based on reducing the error between NN model and PMSM. Different NN strategies have different tracking algorithms and the convergence speeds of different NNs are controllable. In [1], an adaptive NN speed estimator was introduced, while a strategy which uses two NNs was developed in [2] to cooperatively estimate the parameters. However, the stabilities of these NN algorithms are not considered and the robustness cannot be confirmed due to the mismatching of some unidentified and temperature-variation p arameters.

(2) Based on Kalman filters: Kalman filter is one important branch of stochastic filtering techniques which is

Manuscript received May 13, 2009; revised August 10, 2009

This work is supported by China Scholarship Council, National Natural Science Foundation of China (No. 60634020) and the Scientific Researd Foundation of

Education Ministry for the Doctors (No.20060532026).

*Corresponding author. E-mail address: lkan@live.on novel and efficient in parameters identification. Bolognani did deep researches in sensorless drive based on Kalman filter [3], [4]. In [3], a solution to the choice of the EKF covariance is introduced. In [4], a sensorless PMSM drive based on EKF and recursive parameters identification (RPI) is described. RPI is used to design a parameter estimator to overcome the major weak point of sensorless drive based on EKF which is sensitive to the parameter variation. In [5], a simplified reduced-order EKF models for PMSM is developed to estimate its winding resistance and flux-linkage and its computation is significantly reduced due to the reduced order. However, due to the mismatching of unidentified parameters, unconsidering of the robustness and the stability design, normal Kalman filter needs some improvements industrial application, hence, some more advanced robustness filters and robustness design strategy for Kalman filter have been proposed in [6]-[9]. In [6], an $\mathrm{H} \infty$ filter is described and simulation results show that the proposed filter has an automatic increase of bandwidth and potential for more robust performance to poor modelling of disturbances. In [7], P. Bolzern described how to use the $\mathrm{H}$ $\infty$ paradigm to do robustness design for Kalman filter. In [8], John Pearson described a preliminary study for Kalman filter stability by using $\mu$-analysis robust stability technique. Further, the computation of Kalman filter is intensive and usually difficult to implement on-line. Hence, the research focus has been on the reduced-order Kalman filter in order to simplify the algorithm [9].

(3) Based on Model Reference Adaptive System (MRAS): Classical adaptive control can be categor ized into three generations, viz. MIT rule, Lyapunov stability theorem and Popov stability criterion. Without consideration of the stability, MIT rule is simple but not so reliable and has not been considered in practical application although there is still some research. In [10], an estimator based on reducing the output currents error (MIT rule) between PMSM and variable model is proposed and 
parameters such as iron loss resistance, armature resistance, inductance, back EMF, viscous constant and inertia constant are identified. However, there is no stability design in MIT rule and it is not acceptable for practical use. Lyapunov stability theorem is widely used in practical application as the stability design is considered [11]-[13]. In [11], a MRAS design based on Lyapunov theorem is proposed and armature resistance and inductance are identified. However, its design neglects the variation of flux linkage which causes identification error. In [12], an estimator based on Lyapunov theorem is proposed to cooperate with a current decoupling controller, which identifies the stator resistance and inductance successfully. However, a complex computation is required in decoupling control as it needs a more than 10th order closed-loop system and the identification algorithm is efficient only in stringent conditions. In [13], a variable model based on current decoupling is proposed and Lyapunov theorem is employed to design an estimator for identifying stator resistance and inductance. However, the decoupling model needs more computation similar to the problem in [12] and the $\mathrm{d}-\mathrm{q}$ voltages are replaced by the current regulator outputs multiplied by the given gains which cause voltage signal contaminated. In [14], three MRAS estimators are proposed for identifying the rotor speed, stator resistance and rotor-flux magnitude, separately. Routh stability criterion is employed to confirm the stability of the estimators. However, as Routh stability criterion is suit for one dimension single-input-single-output (SISO) system, hence, the design needs equation translation from two-input-two-output system into SISO. In addition, the simultaneous estimation of the stator resistance and the rotor-flux magnitude is impossible. Popov stability criterion can consider the stability and the design of adaptive law can be dictated by its design principles. However, there are few researches based on Popov criterion for PMSM drive [15]-[17] although it is more advanced than the former two theories [18]. In [15]-[17], three similar sensorless PMSM drive systems are proposed and the design satisfies the Popov stability criterion. Although the proposed sensorless drives perform well, the sensitivity to the variable PMSM parameters is still a problem. In [19]-[20], Popov criterion is applied for PMSM parameters identification and good performance has been achieved. In summary, Lyapunov stability theorem is widely used in designing a MRAS estimator due to its simple design process. However, existing developed estimators are currently restricted to identify only one or two parameters and cannot consider the stator resistance and inductance, as well as rotor flux-linkage simultaneously. In addition, as will be detailed in this paper, Popov stability criterion is of better performance in designing a MRAS estimator for PMSM parameter estimation.

In this paper, two schemes of PMSM parameter estimator, based on Lyapunov stability theorem and Popov stability criterion, respectively, are developed and compared. The design of the first proposed new estimator is based on the Lyapunov stability theorem, which is effective in identifying stator resistance, inductance and flux linkage and all the reference model parameters are tracked together. The other improved estimator is based on the Popov stability criterion but with significantly simplified design process compared with that in [20]. It can also successfully estimate the stator resistance, inductance and flux linka ge, while all the reference model parameters in Pop ov strategy are also tracked together. Finally, the relative merits of two estimators are compared by simulation and experiment.

\section{Parameter estimator based on}

\section{Lyapunov stability theorem}

\subsection{Vector control system and design parameters}

In this section, a new estimator based on Lyapunov stability theorem is proposed. The proposed estimators will be applied to a traditional vector control system. In this paper, the state equation of the PMSM is developed based on the dq-axis model in rotor reference frame and the stator currents can be measured. The whole control system is implemented in Matlab/Simulink platform as shown in Fig. 1.

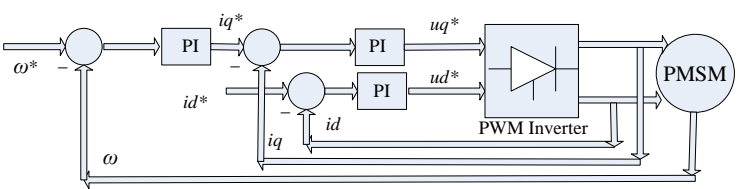

Fig. 1 PMSM vector control in dq-axis reference frame.

The state equation of the unsaturated model of a PMSM can be expressed as (1). Although the parameters in (1) will be varying nonlinearly when the magnet is saturated or the temperature rises, the proposed estimators will work well in tracking the varying parameters.

$$
\begin{aligned}
& \frac{d i_{d}}{d t}=-\frac{R}{L} i_{d}+\omega i_{q}+\frac{u_{d}}{L} \\
& \frac{d i_{q}}{d t}=-\frac{R}{L} i_{q}-\omega i_{d}+\frac{u_{q}}{L}-\frac{\psi}{L} \omega
\end{aligned}
$$

In (1), $i_{d}, i_{q}, u_{d}$ and $u_{q}$ are the dq-axis stator currents and voltages; $\omega$ is the rotor electrical angular speed; $R, L$ and $\psi$ are the stator resistance, dq-axis inductance $\left(L_{d}=L_{q}=L\right)$ and the PMSM flux linkage, respectively. The design parameters and specification of the PMSM in following simulation is listed in Table 1.

Table 1 Design parameters and specification of PMSM

\begin{tabular}{ll}
\hline Rated current & $4 \mathrm{~A}$ \\
Rated speed & $400 \mathrm{rpm}$ \\
DC link voltage & $36 \mathrm{~V}$ \\
Phase resistance & $0.35 \Omega$ \\
d-axis inductance & $0.0027(\mathrm{H})$ \\
q-axis inductance & $0.0027(\mathrm{H})$ \\
Amplitude of flux induced by magnets & $0.075 \mathrm{~Wb}$ \\
Inertia & $0.8 \mathrm{e}-5\left(\mathrm{kgm}^{2}\right)$ \\
Number of poles pairs & 5 \\
\hline
\end{tabular}




\subsection{Structure of variable model and the error state equation}

The PMSM is treated as a reference model and the parameters of variable model will follow the variation of PMSM. ud and uq are the inputs of two models. The errors caused by the differences of id, iq between two models will feed back to the adaptive unit which will adjust the parameters of variable model. The schematic diagram is shown in Fig. 2.

In order to design a stable adaptive system, the equation (1) is transferred into:

$$
\dot{X}=A X+B r+C l
$$

where $A=\left[\begin{array}{cc}-\alpha & \omega \\ \omega & -\alpha\end{array}\right], \quad \alpha=\frac{R}{L}, B=\frac{1}{L}, C=-\frac{\psi}{L}$, $\dot{X}=\left[\begin{array}{l}i_{d} \\ i_{q}\end{array}\right], r=\left[\begin{array}{l}u_{d} \\ u_{q}\end{array}\right], l=\left[\begin{array}{l}0 \\ \omega\end{array}\right]$.

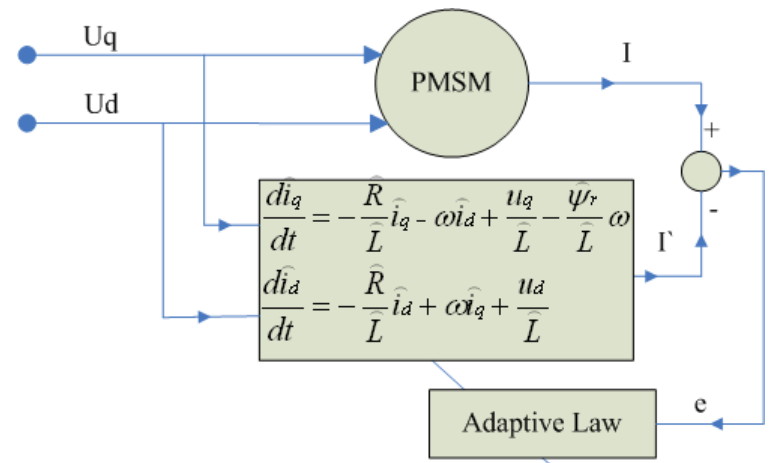

Fig. 2 Model reference adaptive system.

The variable model can be also described as:

$$
\dot{\hat{X}}=\hat{A} \hat{X}+\hat{B} \hat{r}+\hat{C} \hat{l}
$$

Where $\hat{A}=\left[\begin{array}{cc}-\hat{\alpha} & \omega \\ \omega & -\hat{\alpha}\end{array}\right], \hat{\alpha}=\frac{\hat{R}}{\hat{L}}, \hat{B}=\frac{1}{\hat{L}}, \hat{C}=-\frac{\hat{\psi}}{\hat{L}}$,

$$
\hat{X}=\left[\begin{array}{c}
\hat{i}_{d} \\
\hat{i_{q}}
\end{array}\right], r=\left[\begin{array}{l}
u_{d} \\
u_{q}
\end{array}\right], \hat{I}=\left[\begin{array}{l}
0 \\
\omega
\end{array}\right]=l
$$

$u_{d}$ and $u_{q}$ are the inputs of the PMSM and the variable models whose outputs are currents. The inputs are the same but there will be errors between their outputs due to the variation of the parameters of the PMSM.

The errors can be expressed as: $e=X-\hat{X}=\left[\begin{array}{l}e_{1} \\ e_{2}\end{array}\right]$.

Transfer the whole system into error state equation

$\dot{e}=A X-\hat{A} \hat{X}+B r-\hat{B} r+C l-\hat{C} \hat{l}$

$=A X-A \hat{X}+A \hat{X}-\hat{A} \hat{X}+B r-\hat{B} r+C l-\hat{C} \hat{l}$

$=A e+(A-\hat{A}) \hat{X}+(B-\hat{B}) r+h$

Let

$b=B-\hat{B}=\left(\frac{1}{L}-\frac{1}{\hat{L}}\right), a I=A-\hat{A}=(\hat{\alpha}-\alpha) I$,
$h=(C-\hat{C}) l, g_{2}=\frac{\hat{\psi}}{\hat{L}}-\frac{\psi}{L}=C-\hat{C}, \phi^{T}=\left[a, b, g_{2}\right]$,

$s=\left[\begin{array}{lll}\hat{X} & r & l\end{array}\right]^{T}$.

Then, equation (4) can be transferred into equation (5):

$\dot{e}=A e+\phi^{T} S$

Design a Lyapunov function below, which is in the usual positive definite form.

$V(X, t)=\frac{1}{2}\left(e^{T} P e+\phi^{T} \Gamma \phi\right)$

Where $P=\left[\begin{array}{ll}1 & 0 \\ 0 & 1\end{array}\right]=P^{T}, \Gamma=\left[\begin{array}{lll}1 & 0 & 0 \\ 0 & 1 & 0 \\ 0 & 0 & 1\end{array}\right]=\Gamma^{T}$.

$V(X, t)$ is positive definite. Lyapunov stability theorem II is employed here to ensure the global asymptotic stability

of the system and $e=\phi^{T}=0$ is set as the stable

equilibrium point. The theorem is quoted as follows:

1: $V(X, t)$ is positive definite.

2: $\dot{V}(X, t)$ is negative definite.

3: $V(X, t)$ is infinite when $|X| \rightarrow \infty$.

It is obvious that conditions (1) and (3) are achieved and the condition (2) is discussed as follows:

$$
\begin{aligned}
& \dot{V}(X, t)=\frac{1}{2}\left(\dot{e}^{T} P e+e^{T} P \dot{e}+\dot{\phi}^{T} \Gamma \phi+\phi^{T} \Gamma \dot{\phi}\right) \\
& \dot{e}^{T} P e=e^{T} A^{T} P e+s^{T} \phi P e \\
& e^{T} P \dot{e}=e^{T} P(A e)+e^{T} P\left(\phi^{T} s\right) \\
& \dot{e}^{T} P e+e^{T} P \dot{e}=e^{T}\left(P A+A^{T} P\right) e+s^{T} \phi P e+e^{T} P\left(\phi^{T} s\right) \\
& \dot{\phi}^{T} \Gamma \phi+\phi^{T} \Gamma \dot{\phi}=2\left(a \dot{a}+b \dot{b}+g_{2} \dot{g}_{2}\right) \\
& \Rightarrow \dot{V}(X, t)=\frac{1}{2} e^{T}\left(P A+A^{T} P\right) e \\
& +\frac{1}{2}\left(s^{T} \phi P e+e^{T} P\left(\phi^{T} s\right)\right)+a \dot{a}+b \dot{b}+g_{2} \dot{g}_{2} \\
& P A+A^{T} P=-Q=\left[\begin{array}{c}
-2 \alpha \\
-2 \alpha
\end{array}\right]
\end{aligned}
$$

Where $\alpha=\frac{R}{L}>0$, it is obvious that $\frac{1}{2} e^{T}\left(P A+A^{T} P\right) e$ is negative definite.

$\frac{1}{2}\left(s^{T} \phi P e+e^{T} P\left(\phi^{T} s\right)\right)$ 
$=a \hat{i_{d}}\left(i_{d}-\hat{i_{d}}\right)+a \hat{i_{q}}\left(i_{q}-\hat{i_{q}}\right)+b u_{d}\left(i_{d}-\hat{i_{d}}\right)+$

$\left(b u_{q}+\omega g_{2}\right)\left(i_{q}-\hat{i_{q}}\right)$

Therefore,

$\dot{V}(X, t)=\frac{1}{2} e^{T}\left(P A+A^{T} P\right) e+a \hat{i_{d}}\left(i_{d}-\hat{i_{d}}\right)+$

$a \hat{i_{q}}\left(i_{q}-\hat{i}_{q}\right)+b u_{d}\left(i_{d}-\hat{i_{d}}\right)+b u_{q}\left(i_{q}-\hat{i}_{q}\right)+\omega g_{2}\left(i_{q}-\hat{i}_{q}\right)$

$+a \dot{a}+b \dot{b}+g_{2} \dot{g}_{2}$

$=a\left(\hat{i_{d}}\left(i_{d}-\hat{i_{d}}\right)+\hat{i_{q}}\left(i_{q}-\hat{i_{q}}\right)+\dot{a}\right)+b\left(u_{d}\left(i_{d}-\hat{i_{d}}\right)\right.$

$\left.+u_{q}\left(i_{q}-\hat{i}_{q}\right)+\dot{b}\right)+g_{2}\left[\omega\left(\dot{i}_{q}-\hat{i}_{q}\right)+\dot{g}_{2}\right]+$

$\frac{1}{2} e^{T}\left(P A+A^{T} P\right) e$

Then, it is easy to obtain the adaptive law from equation (7).

Let $b\left(u_{d}\left(i_{d}-\hat{i_{d}}\right)+u_{q}\left(\dot{i}_{q}-\hat{i_{q}}\right)+\dot{b}\right)=0$

$a\left(\hat{i_{d}}\left(i_{d}-\hat{i_{d}}\right)+\hat{i}_{q}\left(i_{q}-\hat{i_{q}}\right)+\dot{a}\right)=0$

$\dot{g}_{2}+\omega\left(i_{q}-\hat{i_{q}}\right)=0$

Then it is obvious that $\dot{V}(X, t)$ is negative definite and condition (2) is satisfied. The whole system is therefore global asymptotic stable to converge to equilibrium point.

Therefore, equations (11), (12) and (13) can be achieved from the solutions for equation (8)-(10). Hence, the PMSM resistance, inductance and flux linkage can be estimated and the stability of the whole system can be confirmed.

$$
\begin{aligned}
& a=\hat{\alpha}-\alpha=-\int_{0}^{t}\left[\hat{i}_{d}\left(i_{d}-\hat{i_{d}}\right)+\hat{i}_{q}\left(i_{q}-\hat{i_{q}}\right)\right] d t \\
& \Rightarrow \frac{\hat{R}}{\hat{L}}=\hat{\alpha}=\alpha-\int_{0}^{t}\left[\hat{i}_{d}\left(i_{d}-\hat{i}_{d}\right)+\hat{i}_{q}\left(i_{q}-\hat{i_{q}}\right)\right] d t \\
& b=\frac{1}{L}-\frac{1}{\hat{L}}=-\int_{0}^{t}\left[u_{d}\left(i_{d}-\hat{i_{d}}\right)+u_{q}\left(i_{q}-\hat{i_{q}}\right)\right] d t \\
& \Rightarrow \frac{1}{\hat{L}}=\frac{1}{L}+\int_{0}^{t}\left[u_{d}\left(i_{d}-\hat{i}_{d}\right)+u_{q}\left(i_{q}-\hat{i}_{q}\right)\right] d t
\end{aligned}
$$

$$
\hat{\psi}=\hat{L}\left[\frac{\psi}{L}-\int_{0}^{t} \omega\left(i_{q}-\hat{i}_{q}\right) d t\right]
$$

\section{Parameter estimator based on}

\section{Popov stability criterion}

Compared with the adaptive law design based on Lyapunov stability theorem by which a Lyapunov function is required using designer`s experience, Popov stability criterion is more flexible and designable in obtaining the adaptive law. Using Popov stability criterion for PMSM parameters estimation is firstly described in [19] for resistance and rotor flux estimation. [20] extended the work of [19] and the estimator of inductance was added in [20]. In order to simplify the design process of [19], the positive real lemma is applied in this paper, which does not need complex frequency domain derivation of [20] for ensuring the strictly positive real of feed forward linear model. The main processes of designing adaptive laws based on Popov Theorem can be summarized follows [21]:

(1) Transfer the MRAC system into an equivalent system called nonlinear time variable feedback system, which includes a feed forward linear model and a nonlinear feedback system.

(2) Design part of the adaptive laws which can ensure the nonlinear feedback block satisfies the Popov inequality.

(3) Design the rest part of adaptive laws which ensure the strictly positive real of the feed forward linear model.

(4) Transfer the equivalent system back to MRAC system.

\subsection{Transfer the MRAC system into an equal feedback system}

A typical nonlinear time variable feedback system is drawn in Fig. 3.

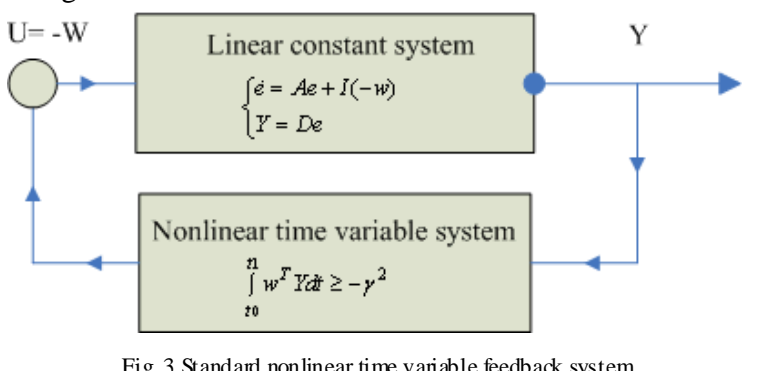

The linear constant system can be derived from error equation (5). Transfer the error equation (5) into the feed forward linear model and the equation can be reformed as equation (14):

$\left\{\begin{array}{l}\dot{e}=A e+I(-w) \\ Y=D e\end{array}\right.$ 
where $I(-w)=(A-\hat{A}) \hat{X}+(B-\hat{B}) U+(C-\hat{C}) G$,

$$
\begin{aligned}
& B-\hat{B}=\left(\frac{1}{L}-\frac{1}{\hat{L}}\right), C-\hat{C}=\frac{\hat{\psi}}{\hat{L}}-\frac{\psi}{L}, \\
& G=\left[\begin{array}{l}
0 \\
\omega
\end{array}\right], U=\left[\begin{array}{l}
u_{d} \\
u_{q}
\end{array}\right]
\end{aligned}
$$

The form of Popov inequality is written below:

$$
\eta\left(t_{0}, t_{1}\right)=\int_{t_{0}}^{t 1} w^{T} y d t \geq-\gamma^{2}
$$

From the foregoing process (2) and equation (14), it can be concluded that the feedback block should satisfy the Popov inequality and the form of inequality can be rewritten as follows:

$$
\eta\left(0, t_{1}\right)=\int_{0}^{t 1} w^{T} y d t=\int_{0}^{t_{1}} w^{T}(D e) d t \geq-\gamma^{2}
$$

\subsection{To ensure the strictly positive real of the feed forward linear model}

As to the strictly positive real of the feed forward linear model, a positive real lemma exists as described below:

(1) A linear constant multi-variable system

$$
\left\{\begin{array}{l}
\dot{x}=A x+B u \\
y=C x+J u
\end{array}\right.
$$

where $(\mathrm{A}, \mathrm{B})$ is controllable and $(\mathrm{A}, \mathrm{C})$ is observable. The transfer function of the system can also be expressed as:

$$
H(s)=J+C(s I-A)^{-1} B
$$

(2) If there is a symmetric positive matrix $\mathrm{P}$, a conventional matrix $\mathrm{K}$ and $\mathrm{L}$, which can confirm the equations below:

$$
\begin{aligned}
& P A+A^{T} P=-L L^{T} \\
& B^{T} P+K^{T} L^{T}=C \\
& K^{T} K=J+J^{T}
\end{aligned}
$$

The system of equation (18) will be positive real and $\mathrm{H}(\mathrm{s})$ will be positive real transfer function.

As to the system of equation (14), the limitation can be simplified as:

$$
\begin{aligned}
& P A+A^{T} P=-Q \\
& I^{T} P=D
\end{aligned}
$$

Here, Q should be symmetrical half positive definite. The transfer function of equation (14) can be described below:

$$
H(s)=D(s I-A)^{-1} I
$$

Substitute $P=\left[\begin{array}{ll}1 & 0 \\ 0 & 1\end{array}\right]$ into equation (19)

$$
P A+A^{T} P=-Q=\left[\begin{array}{cc}
-\frac{2 R}{L} & 0 \\
0 & -\frac{2 R}{L}
\end{array}\right]
$$

$I^{T} P=D=I$

It is also obvious that $(\mathrm{A}, \mathrm{I})$ is controllable and $(\mathrm{A}, \mathrm{D})$ is observable.

As matrix $Q$ is symmetric positive definite (more than half positive definite), it is therefore that the system transfer function of (20) is a strictly positive real transfer function and the feed forward linear model is strictly positive real.

\subsection{Design of adaptive laws}

As the linear compensation matrix D is available, the adaptive law can now be summarized from equation (16). At first, the equation (16) should be decompounded.

$$
\begin{aligned}
& \eta\left(0, t_{1}\right)=\int_{0}^{t 1} w^{T} y d t=\int_{0}^{t 1} w^{T}(D e) d t=\int_{0}^{t 1} w^{T} e d t=\int_{0}^{t 1} e^{T} w d t \\
& =-\int_{0}^{t 1} e^{T}[(A-\hat{A}) \hat{X}+(B-\hat{B}) U+(C-\hat{C}) G] d t \geq-\gamma^{2} \\
& \eta\left(0, t_{1}\right)=-\int_{0}^{t 1} e^{T}(A-\hat{A}) \hat{X} d t-\int_{0}^{t 1} e^{T}(B-\hat{B}) U d t \\
& -\int_{0}^{t 1} e^{T}(C-\hat{C}) G d t
\end{aligned}
$$$$
\geq-\gamma_{1}^{2}-\gamma_{2}^{2}-\gamma_{3}^{2}=-\gamma^{2}
$$$$
\eta_{1}\left(0, t_{1}\right)=\int_{0}^{t_{1}} e^{T}(\hat{A}-A) \hat{X} d t \geq-\gamma_{1}^{2}(a)
$$$$
\eta_{2}\left(0, t_{1}\right)=\int_{0}^{t_{1}} e^{T}(\hat{B}-B) U d t \geq-\gamma_{2}^{2}(b)
$$$$
\eta_{3}\left(0, t_{1}\right)=\int_{0}^{t_{1}} e^{T}(\hat{C}-C) G d t \geq-\gamma_{3}^{2}(c)
$$

We can obtain the adaptive laws separately from the inequalities listed in (a)-(c). As to inequality (a), it can be transferred as follows:

$$
\begin{aligned}
& \eta_{1}\left(0, t_{1}\right)=\int_{0}^{t_{1}} e^{T}(\hat{A}-A) \hat{X} d t= \\
& \int_{0}^{t_{1}}\left(e_{1} \hat{X}_{1}+e_{2} \hat{X}_{2}\right)\left(\frac{R}{L}-\frac{\hat{R}}{\hat{L}}\right) d t \geq-\gamma_{1}^{2}
\end{aligned}
$$

According to the conventional form of adaptive law, simple PI adaptive law can be listed as follows:

$$
-\frac{\hat{R}}{\hat{L}}=-\frac{R}{L}+\int_{0}^{t} G_{1}(\tau) d \tau+G_{2}(\tau)
$$


$\Rightarrow \eta_{1}\left(0, t_{1}\right)=\int_{0}^{t 1}\left(e_{1} \hat{X}_{1}+e_{2} \hat{X}_{2}\right)\left(\frac{R}{L}-\frac{R}{L}+\int_{0}^{t} G_{1}(\tau) d \tau\right.$

$\left.+G_{2}(\tau)\right) d t$

$$
=\int_{0}^{t 1}\left(e_{1} \hat{X}_{1}+e_{2} \hat{X}_{2}\right)\left(\int_{0}^{t} G_{1}(\tau) d \tau+G_{2}(\tau)\right) d t=\geq-\gamma_{1}^{2}
$$

It can also be decompounded into two parts:

$$
\begin{aligned}
& \eta_{11}\left(0, t_{1}\right)=\int_{0}^{t 1}\left(e_{1} \hat{X}_{1}+e_{2} \hat{X}_{2}\right)\left(\int_{0}^{t} G_{1}(\tau) d \tau\right) d t \geq-\gamma_{11}^{2} \\
& \eta_{12}\left(0, t_{1}\right)=\int_{0}^{t_{1}}\left(e_{1} \hat{X}_{1}+e_{2} \hat{X}_{2}\right) G_{2}(\tau) d t \geq-\gamma_{12}{ }^{2}
\end{aligned}
$$

Since

$\int_{0}^{t} f(t) \dot{f}(t) d t=\frac{1}{2}\left[f^{2}(t)-f^{2}(0)\right] \geq-\frac{1}{2} f^{2}(0)$

$\int_{0}^{t} f^{2}(t) d t \geq 0$

It is easy to obtain G1 and G2. The adaptive law of $\frac{\hat{R}}{\hat{L}}$ can be easily obtained:

$$
\frac{\hat{R}}{\hat{L}}=\frac{R}{L}-K_{0} \int_{0}^{t}\left(e_{1} \hat{X}_{1}+e_{2} \hat{X}_{2}\right) d t-K_{2}\left(e_{1} \hat{X}_{1}+e_{2} \hat{X}_{2}\right)
$$

Then the adaptive laws for other parameters can be easily obtained by using the same computation principle and are listed below:

$$
\begin{aligned}
& \frac{1}{\hat{L}}=\frac{1}{L}+K_{1} \int_{0}^{t}\left(u_{d}\left(i_{d}-\hat{i_{d}}\right)+u_{q}\left(i_{q}-\hat{i_{q}}\right)\right) d t \\
& +K_{2}\left(u_{d}\left(i_{d}-\hat{i_{d}}\right)+u_{q}\left(i_{q}-\hat{i}_{q}\right)\right) \\
& \frac{\hat{\psi}}{\hat{L}}=\frac{\psi}{L}-K \int_{0}^{t} \omega(i q-\hat{i} q) d t-K 2 \omega\left(i q-\hat{i_{q}}\right)
\end{aligned}
$$

It is obvious that the foregoing adaptive laws are similar with the adaptive law based on Lyapunov Theorem. This is due to the fact that both Popov criterion and Lyapunov theorem need the positive real character for the transfer function of error model. Therefore, their general forms of adaptive laws are similar, too. However, due to the adaptive law based on Popov criterion is more designable and flexible, it seems more satisfying to use Popov criterion in MRAC design.

\section{Simulation results}

In order to compare and verify the performance of the proposed two estimators, with the same integral gain they are applied to the vector control system of the PMSM,
Fig.1, whose design parameters are given in Table 1. Fig.5 shows the simulated tracking performance of two estimators based on Matlab/Simulink platform. Both the estimators perform very well. As seen from Fig.5, although the parameters, such as stator resistance, inductance and flux linkage vary from their normal values to $50 \%$ together, the robustness of the estimators against the uncertainty of variation is still excellent and the estimators will converge to their stable working points (error=0) due to the global asymptotic stability design and super stability design, respectively.

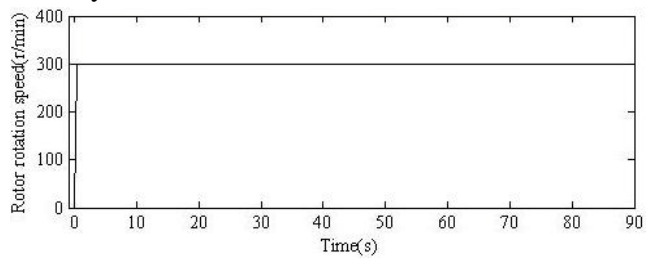

Fig.4 Actual rotor rotation speed.
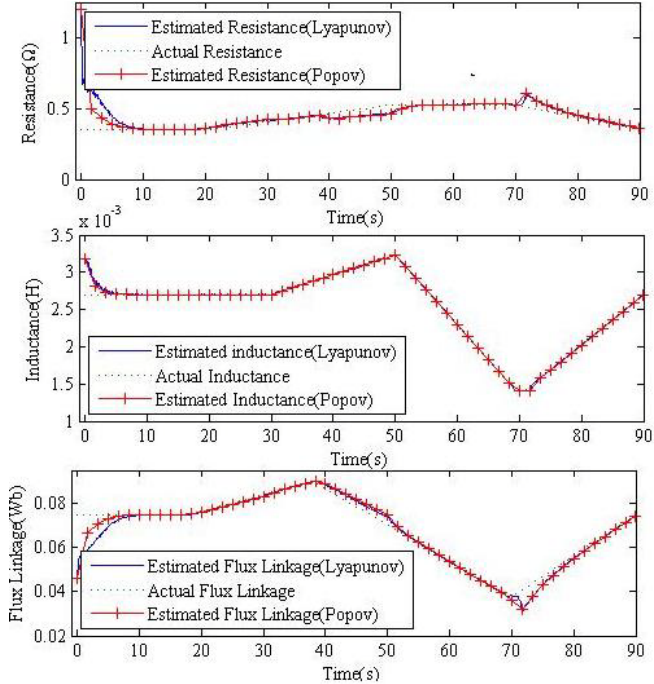

Fig. 5 Actual and estimated resistances, inductance and flux linkage.
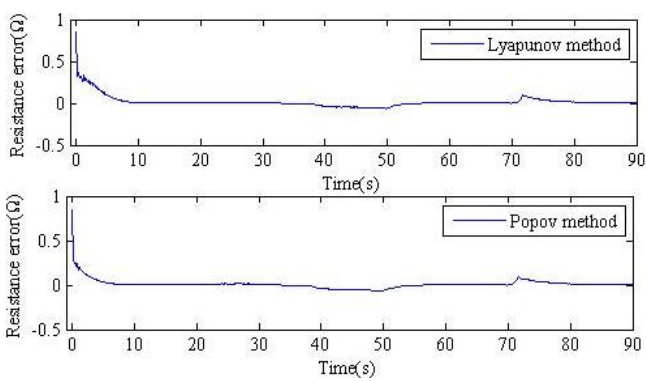

Fig.6 Comparison of resistance identification dynamic errors between Lyapunov method and Popov method.
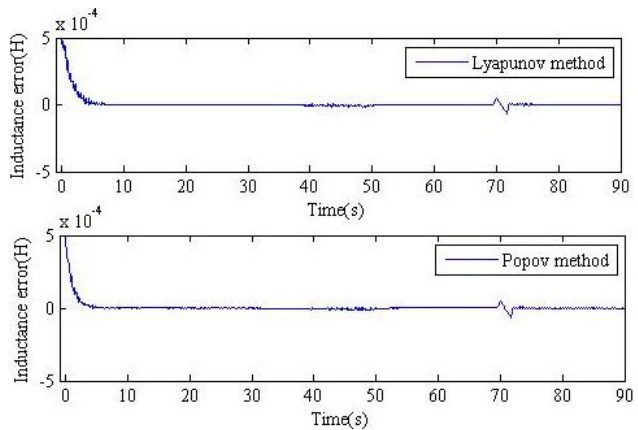
Fig.7 Comparison of inductanceidentification dynamic errors between Lyapunov method and Popov method.

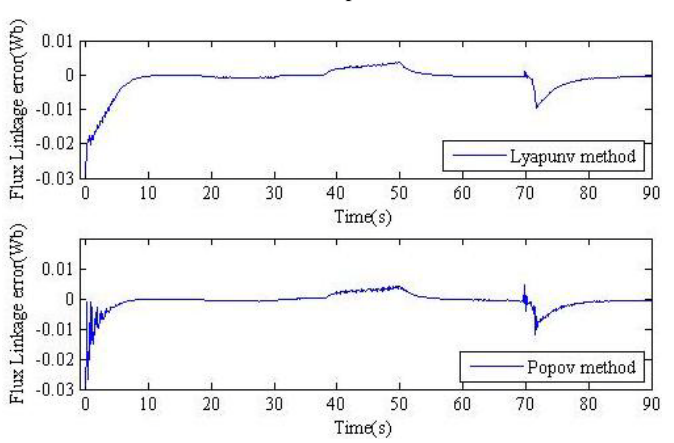

Fig.8 Comparison of flux linkage identification dynamic errors between

$$
\text { Lyapunov method and Popov method. }
$$

As can be seen from Figs. 6-8, the relative errors of both estimators converge to zero. They do not have too much difference in simulated performance as fixed constant parameters are used. However, as will be shown in the following section, their performance will be different in practical experiments due to parameter variation.

\section{Experimental verification}

The performance of the proposed estimators is further verified experimentally on a DSP (TMS TI 2812) based PMSM drive system. The design parameters of the PMSM are given in Table 1 and the DSP sampling period is 0.08333 ms. The test rig setup is shown in Fig. 9. The measured DC link voltage is used to obtain the actual stator voltages while the currents are obtained from the current sensors. The waveform of rotor rotation speed, dq-axis currents and voltage are shown in Fig. 10. The experimental data are sampled and filtered by low-pass Butterworth filter for identifying the parameters.
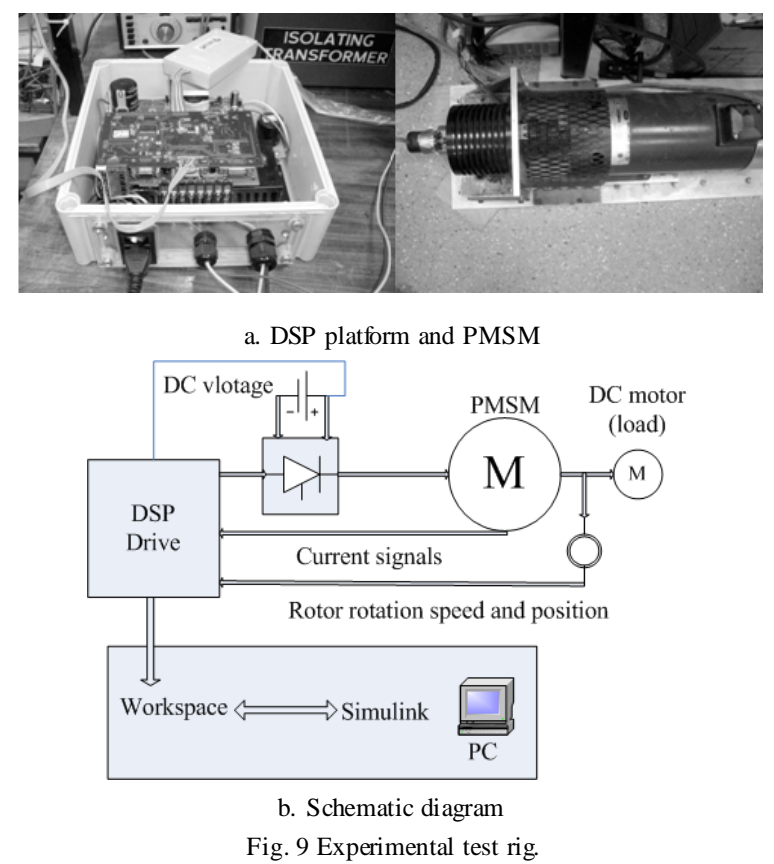

An equation for discrete integral computation is shown as follows:

$$
\left\{\begin{array}{l}
y(k+1)=y(k)+K I * T s * x(k) \\
\text { Step }=K I * x(k) * T s
\end{array}\right.
$$

Equation (24) is the conventional method to carry out the discrete integral computation in DSP. KI is the integral gain of the discrete PI controller and Step is considered to be the discrete integral step length. Therefore, it is evident that the estimated values such as flux linkage, inductance and resistance are sensitive to Step. Fig. 11 depicts the estimation result based on Popov method which exhibits excellent performance. Fig. 12 depicts the estimation result based on Lyapunov method.

As overshooting and integral saturation may occur due to the use of too large integral gain and the convergence speed cannot be ensured if too small integral gain is set, the integral gain for resistance adaptive law is experimentally determined to be 2 in Figs. 11 and 12. As can be seen from Fig. 11, although proportional unit in Popov method may cause a transient overshoot at the beginning, it can offer better response speed. Fig. 12 shows that Lyapunov method has a trade-off between the convergence speed and the integral saturation. Therefore, the estimator based on Ly apunov has poor performance in fast tracking.

In summary, although in simulation there is no significant difference between the estimators based on Lyapunov method and Popov method, the performance of the two methods is different in practical application due to parameter variation. Therefore, although various Lyapunov method based MRAS estimators have been used for identifying one or two parameters [11]-[13] due to its easy design process, it is evident that the estimator based on Popov method exhibits better performance in practice. It is preferable to use Popov method in MRAS design.

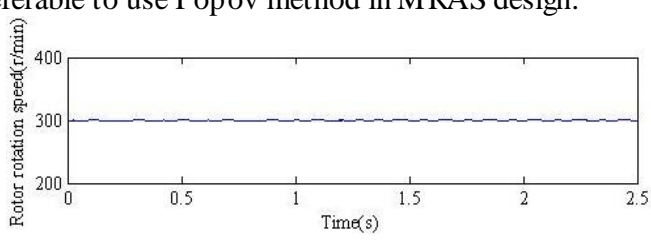

(a) Rotor rotational speed
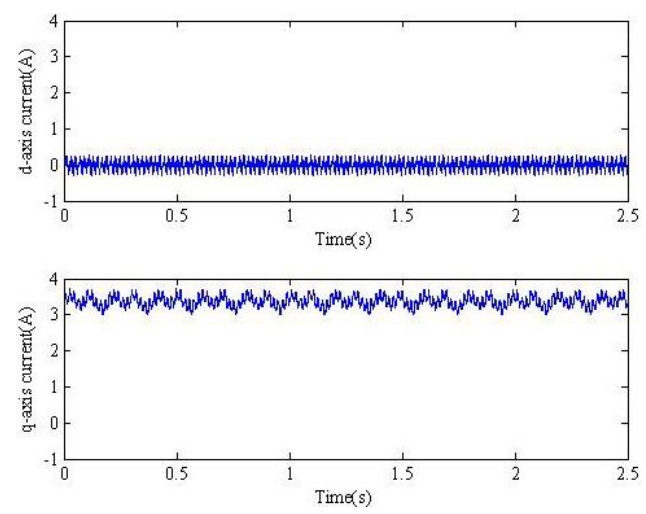

(b) Current 

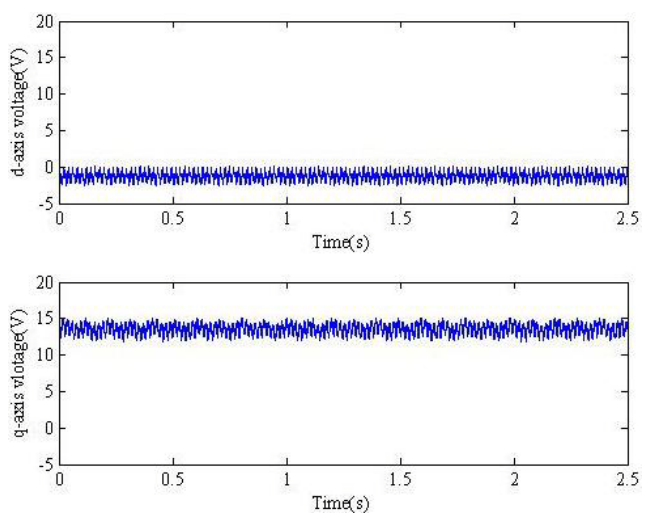

(c) Voltage

Fig. 10 Measured rotor speed, dq-axis currents, voltages.
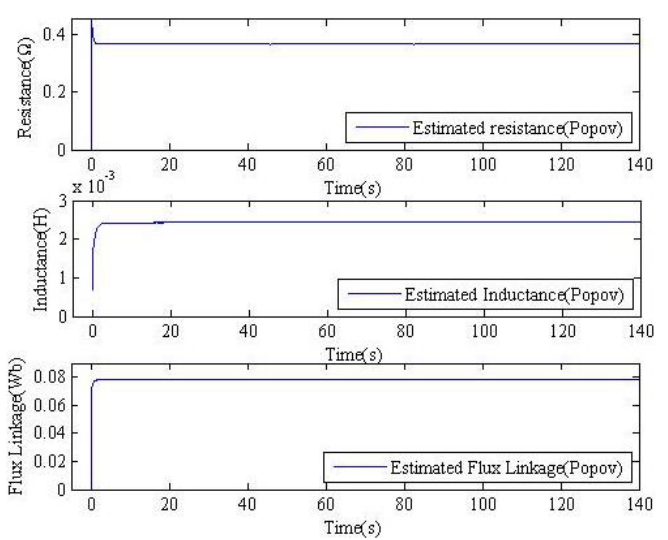

Fig. 11 Estimated resistance, inductance and flux-linkage by Popov method.
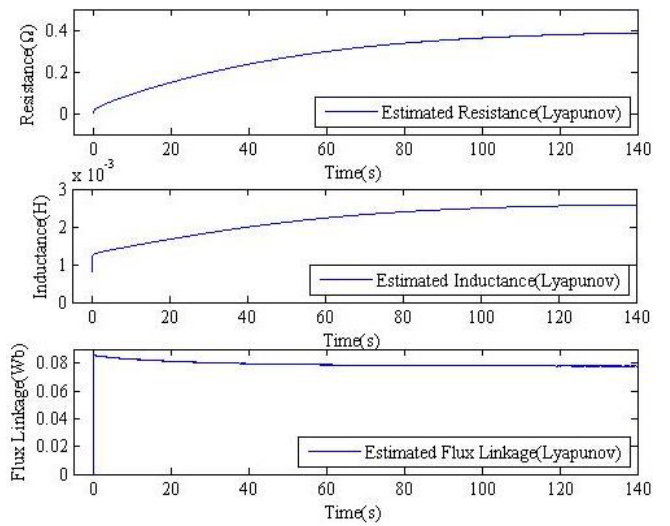

Fig. 12 Flux-linkage and inductance estimated by Lyapunov method.

\section{Conclusions}

Two PMSM parameter estimators based on Lyapunov stability theorem and Popov stability criterion, respectively, are developed and compared. Both are effective in tracking the motor parameters. As all the parameters in (1) are identified and the stability of the system is ensured, the simulation and experimental results show good performance in robustness and accurate tracking. As three parameters ( $\mathrm{R}, \mathrm{L}$ and $\psi$ ) in (1) may vary due to magnetic saturation and temperature rise, it is necessary to simultaneously estimate three parameters otherwise the estimation result will suffer from the mismatching of unidentified parameter. For example, in [11], the resistance is correctly estimated but the author found that the inductance could not be correctly estimated by experiment due to the offset of unidentified parameters. The two estimators developed in this paper can both estimate three parameters simultaneously. In addition, the two estimators do not need too much computation as there is only one variable model needed to compute and three PI (or integral) computations. The experimental results also verify that the Popov method, which can overcome the parameter variation in a practical system, exhibits better performance than Lyapunov method. Furthermore, the processes of designing a controller based on the Popov criterion do not need the expert experience to design a Lyapunov function and the adaptive law is designable via changing the function satisfying the Popov integral inequality. The relevant researches such as robustness design for adaptive estimators, the inverter nonlinearity compensation [22] for stator resistance estimation and estimators for salient-pole PMSM, etc. are being further investigated and will be reported in another paper.

\section{References}

[1] M.A. Rahman, and M.A. Hoque, "On-line adaptive arti ficial neural network based vector control of permanent magnet synchronous motors," IEEE Trans. on Energy Conversion, vol. 13, no. 4, pp. 311-318, Dec. 1998.

[2] T. Liu, M. Elbuluk, and I. Husain, "Sensorless adaptive neural network control of permanent magnet synchronous motors," Proc. Electric Machines and Drives, 1999, pp. 287-289.

[3] S. Bolognani, L. Tubiana, and M. Zigliotto, "Extended Kalman filter tuning in sensorless PMSM drives," IEEE Trans. on Ind. Applications, vol. 39, no. 6, pp. 1741-1747, Nov.-Dec. 2003.

[4] S. Bolognani, M. Zigliotto, and K. Unterko fler, "On-line parameter commissioning in sensorless PMSM drives," In Proc. of the IEEE International Symposium on Industrial Electronics, Guimaraes, Portugal, vol. 2, pp.480 - 484, July 1997.

[5] Z.Q. Zhu, X. Zhu, and P.D. Sun, “Estimation of winding resistance and PM flux-linkage in brushless AC machines by reduced-order extended Kalman Filter,” In Proc. 2007 IEEE International Conference on Networking, Sensing and Control, London, U.K., pp. 740-745, 2007.

[6] H. Tsaknakis, and M. Athans, "Tracking maneuvering targets using $\mathrm{H} \infty$ filters,” In Proc. American Control Conference, Baltimore, U.S.A., vol. 2, pp. 1796-1803, 1994.

[7] P. Bolzern, P. Colaneri, and G. De Nicolao, "Robustness in adaptive filtering: How much is enough?" In Proc. of the $36^{\text {th }}$ IEEE Conf. on Decision and Control, San Diego, U.S.A., vol. 5, pp.4677-4679, 1997. [8] J. Pearson, R. Goodall, M. Eastham, and C. MacLeod, "Investigation of Kalman filter divergence using robust stability techniques [combat aircraft tracking/navigation system]," In Proc. of the $36^{\text {th }}$ IEEE Conf. on Decision and Control, San Diego, U.S.A., vol. 5, pp. 4892-4893, 1997.

[9] X. Zhu, Z.Q. Zhu, and David Howe, “Application of full-order and simplified EKFs to sensorless PM brushless AC machines,” International Journal of Automation and Computing, vol. 2, no. 2, pp. 179-186, Dec. 2005.

[10] T. Senjyu, K. Kinjo, N. Urasaki, and K. Uezato, "Parameter measurem ent for PMSM using adaptive identification," In Proc. of the 2002 IEEE International Symposium on industrial electronics, L`Aquila, Italy, vol. 3, pp. 711-716, 2002.

[11] L. Liu, and D.A. Cartes, "Synchronisation based adaptive 
parameter identification for permanent magnet synchronous motors," IET Proceedings-Control Theory \& Applications, vol. 1, no. 4, pp. 1015-1022, 2007.

[12] B. N. Mobarakeh, F. Meibody-Tabar, and F.M. Sargos, "On-line identification of PMSM electrical parameters based on decoupling control," Conf. Rec. of the IEEE Industrial Applications Society Annual Meeting, Chicago, U.S.A., vol. 1, pp. 266-273, 2001.

[13] T. Boileau, B. Nahid-Mobarakeh, and F. Meibody-Tabar, "On-line identification of PMSM parameters: model-reference vs. EKF,” In Proc. IEEE Industry Applications Society Annual Meeting, Edmonton, Canada, pp. 1-8, 2008.

[14] M. Rashed, P.F.A. Macconnell, A.F. Stronach, and P. Acarnley, "Sensorless indirect-rotor-field-orientation speed control of a permanent-magnet synchronous motor with stator-resistance estimation," IEEE Trans. on Industrial Electronics, vol. 54, no. 3, pp. 1664-1675, 2007.

[15] M.H. Park, and H.H. Lee, "Sensorless vector control of permanent magnet synchronous motor using adaptive identification," In Proc. IEEE 15th Annual Conference of Industrial Electronics Society, Philadelphia, U.S.A., vol. 1, pp. 209-214, 1989.

[16] D. Xu, and Y. Gao, "An approach to torque ripple compensation for high performance PMSM servo system," In Proc. IEEE 35th Annual Power Electronics Specialists Conference, Aachen, Germany, vol. 5, pp. 20-25, 2004.

[17] H.M. Kojabadi, and L. Chang, "Sensorless PMSM drive with MRAS-based adaptive speed estimator," In Proc. IEEE 37th Power Electronics Specialists Conference, Jeju, Korea, pp. 1-5, 2006.

[18] W.M. Haddad, and D.S. Bernstein, "Parameter-dependent Lyapunov functions and the Popov criterion in robust analysis and synthesis," IEEE Transactions on Automatic Control, vol. 40, no. 3, pp. 536 - 543, 1995.

[19] Kyeong-Hwa Kim, Se-Kyo Chung, Gun-Woo Moon, In-Cheol Baik and Myung-Joong Youn, "Parameter estimation and control for permanent magnet synchronous motor drive using model reference adaptive technique," In Pro. IEEE 21st International Conference on Industrial Electronics, Control, and Instrumentation, Orlando, U.S.A., vol. 1, pp. 387-392, 1995.

[20] Quntao An and Li Sun, "On-line parameter identification for vector controlled PMSM drives using adaptive algorithm," In Pro. IEEE Vehicle Power and Propulsion Conference, Harbin, China, pp. 1-6, Sept. 2008.

[21] D. Zhou, "Introduction to adaptive control of non-linear systems," Tsinghua University Publication\& Springer, China, 2002.

[22] J.W. Choi, and S.K. Sul, "Inverter output voltage synthesis using novel dead time compensation," IEEE Trans. on Power Electronics, vol. 11, no. 2, pp. 221-227, 1996.

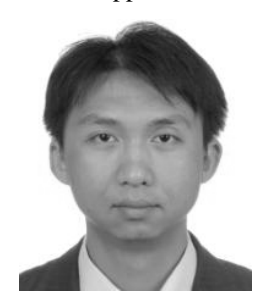

Kan Liu received his BEng degree in automation from the Hunan University, China, in 2005. Then, he started his courses for a joint Master/PHD degree in Hunan University. In 2008, he was supported by China Scholarship Council to go on his study as a joint PHD student/visiting student at the University of Sheffield, U.K. Currently, he is working in the Department of Electronics and Electrical Engineering at the University of Sheffield. His research interest focuses on brushless AC motor parameters estimation by control theory.

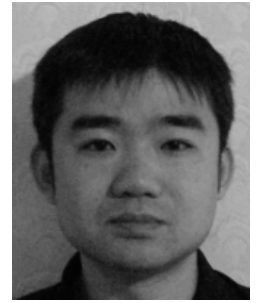

Qiao Zhang received his BEng and M.SC degrees in Department of Control Science and Technology from Huazhong University of Science and Technology, China, in 2003 and 2006. In 2008, he was supported by China Scholarship Council to go on his study as a joint PHD student/visiting student at the University of Sheffield, U.K. Currently, he is working in the Department of Electroni cs and Electrical Engineering at the University of Sheffield. His research interest focuses on brushless AC motor parameters estimation and machine drives

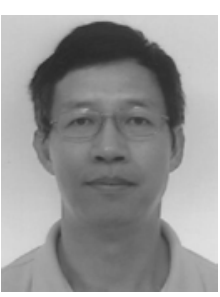
design.

Z Q. Zhu received the B.Eng. and M.Sc. degrees in electrical and electronic engineering from Zhejiang University, Hangzhou, China, in 1982 and 1984, respectively, and the Ph.D. degree in electrical and electronic engineering from the University of Sheffield, Sheffield, U.K., in 1991. From 1984 to 1988, he was a Lecturer with the Department of Electrical Engineering, Zhejiang University. Since 1988, he has been with the University of Sheffield, where he was initially a Research Associate and was subsequently appointed to an established post as Senior Research Offi cer/Senior Research Scientist. Since 2000, he has been a Professor of Electrical Machines and Control Systems with the Department of Electronic and Electrical Engineering, University of Sheffield, and is currently Head of the Electrical Machines and Drives Research Group. His current major research interests include design and control of permanent magnet brushless machines and drives, for applications ranging from automotive, aerospace, to renewable energy.

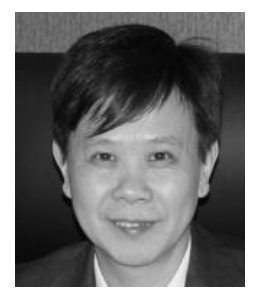
He is a Fellow of IEEE.

Jing Zhang received his BEng, M.Sc. and PHD degrees from Hunan University, China, in 1982, 1984 and 1997, respectively. His research interests in optimal control, fuzzy control and intelligent control of rotary kiln. He has published more than 100 papers on journals and conferences and was awarded China national second prize of scienti fic and technological progress.

Anwen Shen received his BEng and M.SC degrees in Department of Electrical Engineering in Zhejiang University and PHD degree in Huazhong University of Science and Technology. Currently, he is a professor in Department of Control Science and Technology of Huazhong University of Science and Technology His research interest focuses on machine drives design, PWM strategies, intelligent control and progress control.

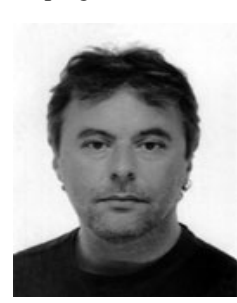

Paul Stewart Professor Paul Stewart is the chair of Aeronautical and Automotive Engineering, School of Computing, Science and Engineering and the president of the IEEE United Kingdom and Republic of Ireland Industrial Electronics Chapter. He is now working in the University of Salford. He has published over 40 papers in the field of control theory applications, electromechanical motion control, power systems, multi-objective optimization and intelligent systems. 